\title{
An Unusual Case of Adrenal Metastases
}

M Alhelfi, L Nelson, E Baker, A Mukherjee, A Robinson.

Department of Endocrinology, Salford Royal Foundation Trust Hospital, Salford. M6 8HD

\section{Case Scenario}

73-year old gentleman referred by GP with a two month history of worsening dizziness, malaise, postural hypotension, general deterioration and confusion. A swinging pyrexia ranging from $38-40^{\circ} \mathrm{C}$ was evident over 3 weeks. PMH of NSCLC T2b N0, treated with left lower lobectomy \& chemotherapy 3 years prior.

\section{Investigations, Management and Progress}

- At presentation his CT TAP showed bilateral bulky adrenal glands but no other abnormality. The patient's bloods showed mild raised inflammatory markers.

-An SST test was done as suspicion of adrenal insufficiency which revealed flat response.

-All microbiology cultures were clear including several sets of blood cultures. The patient's CXR, viral screen and autoimmune screen were normal and echocardiogram showed normal LV function with no valvular lesions. The LP results revealed mild raised CSF protein but PCR for TB as well as flow cytometry/immunophenotyping were all negative.

- He was treated for sepsis of unknown origin with antibiotics, acyclovir and fluids as well as high dose hydrocortisone.

- No significant improvement was observed.

- Repeat CT-TAP showed progressive, generalized enlargement of both adrenal glands with peri-adrenal inflammatory change.

- CT guided adrenal biopsy showed needle cores of non-necrotic fibrovascular tissue and some adipose tissue, widely infiltrated by poorly differentiated malignant cells.

-Whilst an inpatient, despite all supportive care, progressive, inexorable deterioration occurred. The patient was transferred to medical HDU as he deteriorated further. In spite of best supportive care he continued to spike fevers and died following cardiac arrest.

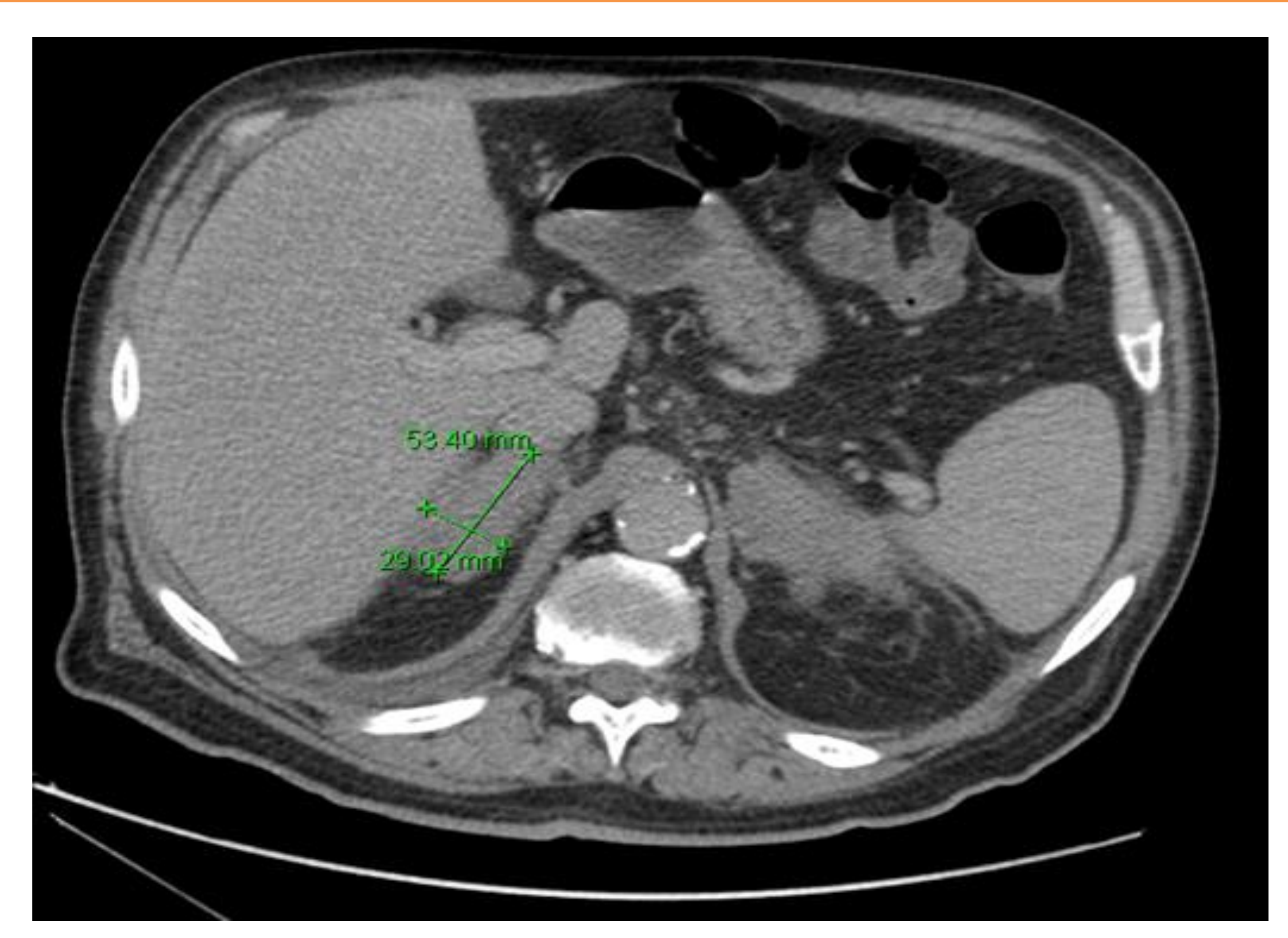

Figure 1. MRI adrenals

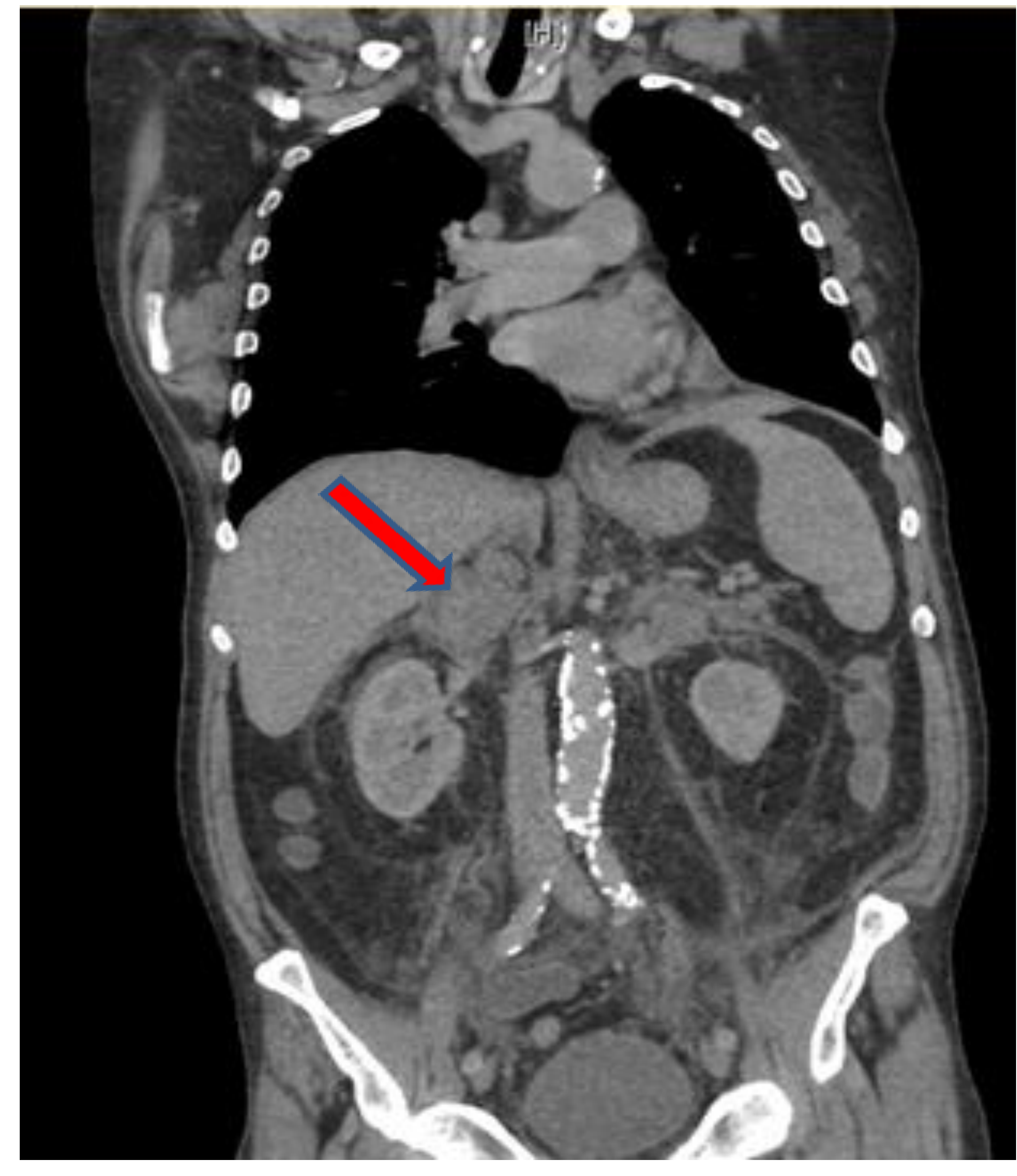

Figure 2. MRI appearance of Rt adrenal

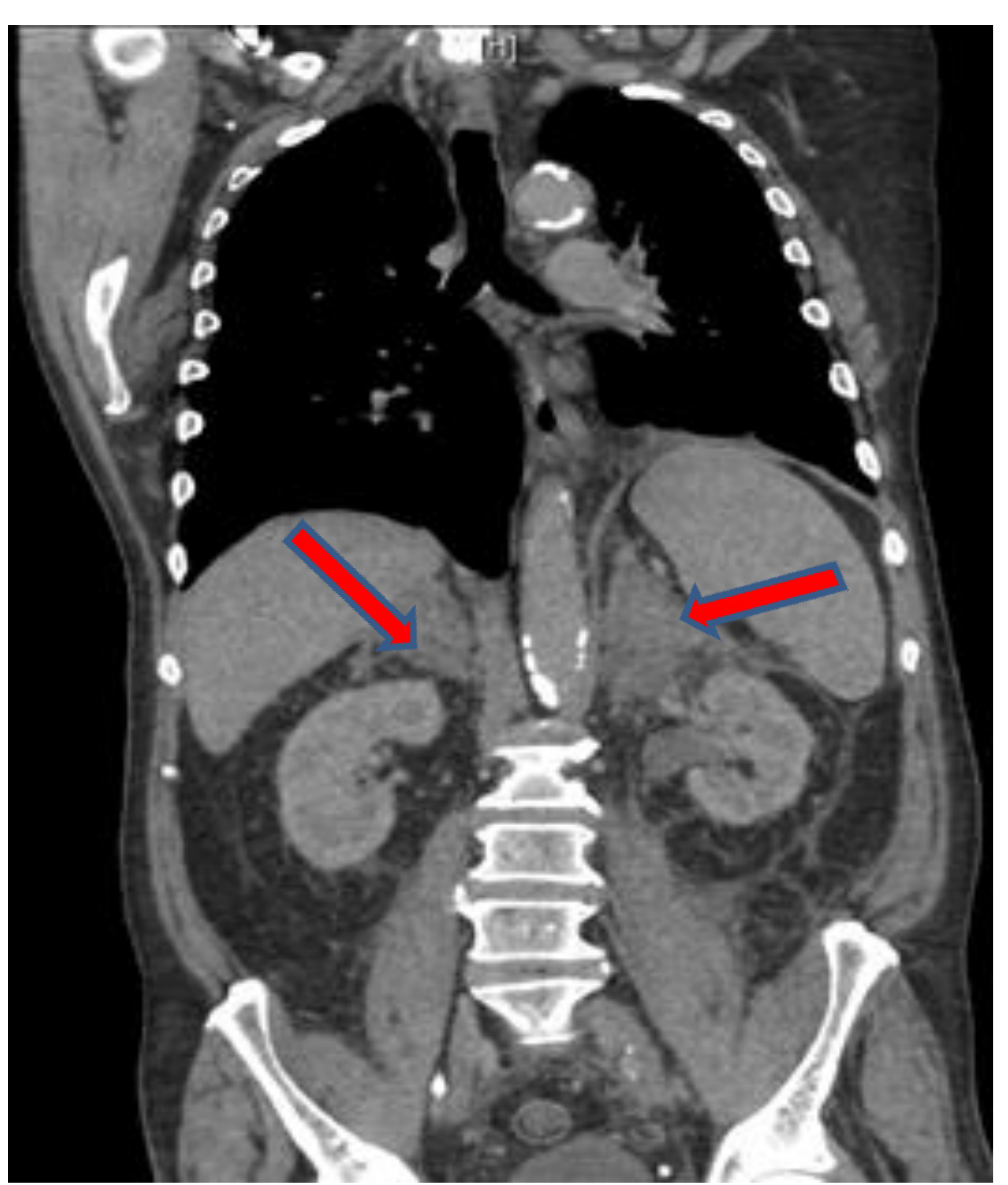

Figure 3. MRI appearance of both adrenal

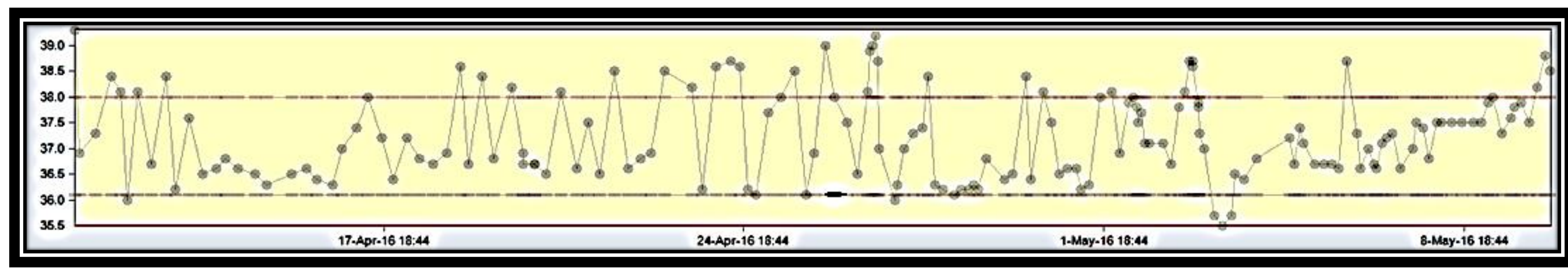

Figure 4. Temperature graph

\section{Post-mortem}

-PATHOLOGICAL SUMMARY : At autopsy examination, both the adrenals were replaced by a mass lesion, which on histology showed high grade B-cell lymphoma. The malignant cells were also seen involving other organs including brain, kidneys, liver and lungs, predominantly within the blood vessels.

Death was due to;

la) DISSEMINATED HIGH GRADE B-CELL LYMPHOMA

\section{Conclusions}

- The patient presented with rapidly progressive adrenal insufficiency associated with swinging pyrexia and malignant adrenal infiltration, without necrosis or other evidence of metastatic disease or positive microbiology. An atypical presentation of adrenal metastasis was the final ante-mortem working diagnosis.

- Disseminated high grade B-cell lymphoma involving the adrenal glands was the post-mortem cause of death.

\section{Points for Discussion}

- Development of adrenal failure requires destruction of more than $90 \%$ of the adrenal gland tissue. Most cases of adrenal insufficiency caused by malignant neoplasms are due to metastases from the lung, breast or stomach or malignant melanoma.

- Non- Hodgkin lymphoma (NHL) arising in the endocrine system represents only 3\% of extranodal malignant lymphomas, and is usually confined to the thyroid gland.

- About 100 cases of adrenal lymphoma have been reported to date, with bilateral involvement in $70 \%$. Primary adrenal lymphoma tends to occur more commonly in males (2.5-7 times), with an average age of 61 years (38-81). Morphology is more commonly of a large cell lymphoma with a B cell phenotype. Symptoms may occur due to the tumour itself or to adrenal failure.

\section{Reference}

Venizelos, I et al. (2007). High grade primary adrenal intravascular large B-cell lymphoma manifesting as Addison disease.. Rev Esp Enferm Dig.. 99 (8), 471-4. 